thelial tumor cells. A tumor examined showed in light microscopy a rich net work of vascular vessels. Electron microscopic fields were distinguisched also by a multitude of blood vessels, comprized mostly of capillaries. The cell membrane of the neoplastic cells was occasionally directly in contact with the basement membrane. In this case intercellular spaces were sepa. rated widely, which contained occasionally erythrocytes. These spaces were seen enclosed by neoplastic cells with prominent attachment plates. No evidences, however, were demonstrated, indicating the tumor cell forming basement membrane. In areas where the light microscopy presented a pseudoxanthomatous appearance, tumor cell cytoplasms were filled with vacuoles, containing amorphous materials of lipid in nature. It seems likely that the vacuoles develope from pinocytic vesicles ingesting lipid materials. A tumor was seen in light microscopy including a large number of psammoma bodies. In electron microscopy they were composed of masses of collagenous fiber bundles with an background of amorphous materials. Electron dense materials of calcium in nature were found occasionally replacing the fiber bundles. This mass was surrounded by long curving cytoplasmic processes containing abundant dense granules. It is likely that the psammoma body is formed by degeneration in the center of the whorls and subsequent fibroblastic activity of encircling tumor cells. It reqires, however, further study to draw conclusions to the precise mode of psammoma body formation.

\title{
S-A-4. The study on Fatty acid in Cerebroespinalfluid
}

\section{(Gas-chromatographycal analysis)}

Isamu YANAGISA WA

Department of Biochemistry, School of Medicine, Toho University

Koreyoshi Makino, Tetsuro Miwa, Kozaburo Kimura, Iwao IWAKI and Yoshihisa ONODERA

Department of Surgery, Tokyo Medical College

Cerebrospinalfluid-fatty acid in clinical cases was analyzed by means of Gas-chromatography.

On this study, Hitachi-KGL-2B FID 2 type Gas-chromatography was used usually, and Gas-chromatography condition was as following;

1) Column: $3 \mathrm{~m} \times 3 \mathrm{~m}$. 
Diasolid S, 80-100 mesh, 10\% Polydiethylenglycolsuccinate.

2) Carrier gas: Herium $1.7 \mathrm{Kg} / \mathrm{cm}^{2}$.

3) Columntemperature : $200^{\circ} \mathrm{C}$

4) Sample temperature: $270-300^{\circ} \mathrm{C}$

$50 \mathrm{mg} / \mathrm{dl}$ of Arachidic acid to be soluble in Methanol as standard, was added to the sample ( $4 \mathrm{ml}$ of CSF), and after saponifing, the sample was analyzed. The quantitative error of the recovery test for Arachidic acid was lesser than $\pm 5 \%$.

\title{
Result
}

1) normal group

The constitutions of fatty acids in CSF were remarkably different from that in serum. The fatty acids, has lesser carbon number than Palmitic were few in serum, and much in CSF.

On the contrary, the fatty acids, range over C16, were of large quantity in serum, and few in CSF.

It was found that an unkown fatty acid existed between Palmitic acid and Myristic acid in CSF.

2) brain tumor group.

Qualitatively, the constitutions of CSF-fatty acids were the same as that in normal CSF. However the quantity of CSF-fatty acids was diminished.

\section{S-A-5. Lipid and it's Fatty Acid Composition of Brain Tumors}

\author{
Yasushi Tatsuzawa, Masahiro Kato, Tsuneo Hasegawa \\ and Toyozo Aizawa \\ Department of Internal Medicine, Keio University \\ Shoji IsHIMORI \\ Department of Surgery, Keio University
}

By the methods of thin-layer chromatography, gas-liquid chromatography and biochemical assay, the lipid and it's fatty acid composition were analyzed in the following materials; glioblastoma multiforme (solid and soft area), astrocytoma, oligodendroglioma, two cases of meningioma, neurofibroma, craniopharyngioma and also grey and white matter as the controls.

Phospholipid content of tumors showed lower level than that of normal grey matter, but no significant differences were found among tumors. 